\title{
Performance Evaluation of Fuzzy based DCP and AHE for Underwater Image Haze Removal
}

\author{
Sonali Talwar \\ Research Scholar, Department of Computer \\ Science and Engineering \\ DAVIET, Kabir Nagar, Jalandhar-144001, Punjab, \\ India
}

\begin{abstract}
Underwater images are used to explore the unique life and the world that exists under the water. These images have less clarity, diminishing colors, low contrast .All these issues are a result of haze in underwater images. So, Underwater image haze removal algorithms become very important and necessary for many vision applications. In this paper, after a brief overview of existing methods of haze removal and discovering their limitations, we present a novel technique of underwater image haze removal based on Fuzzy Based DCP and AHE. AHE has been used to remove the problem of uneven illumination. The proposed technique has the ability to remove the limitations of existing techniques. Different kind of image quality assessment metrics have been used to evaluate the effectiveness of proposed technique over the existing one. The proposed technique give efficient results for different hazy underwater images by removing the haze to a good extent and improving the quality.
\end{abstract}

\section{Keywords}

Underwater Image Haze Removal, Fuzzy Logic

\section{INTRODUCTION}

Underwater images are captured to explore underwater world, for applications such as Underwater Archaeology, Weather Forecasting, Underwater robotics [5], Seismic monitoring for oil extraction by underwater sensor networks [8], Remote Sensing, Deep Sea Diving and underwater aquatic life. The underwater images have problems such as limited range of visibility, low contrast, non-uniform lighting and illumination, bright artifacts, noise, blurring, and diminishing color. These are caused by factors such as: Scattering is caused by large suspended particles such as turbid water [24] which result in blurring of image features. Backscattering result in the loss of contrast between object and its surroundings generating a characteristic veil that superimposes itself on the image and hides the scene [11]. Color change corresponds to varying degrees of attenuation encountered by light travelling in water with different wavelengths rendering water dominated by a bluish tone [24]. But the biggest problem in underwater images is haze. Haze degrades scene contrast and result in diminishing of colors. Underwater Image Haze Removal strengthens and improves the performance of various vision algorithms, increase the visibility of the scene, improves the contrast and quality of underwater images. Various underwater image enhancement techniques [13], [14], [15] have also been developed by researchers to improve the quality and contrast of underwater images.

\author{
Rajesh Kochher \\ Assistant Professor \\ Department of Information Technology \\ DAVIET, Kabir Nagar, Jalandhar-144001, Punjab, \\ India
}

\subsection{Dark Channel Prior (DCP)}

The Dark Channel prior is a kind of statistics of the haze free outdoor images. It is based on a main thought that most of local patches in haze free outdoor images contain pixels which have very low intensities in atleast one color channel (RGB) called dark pixels [12]. In the haze image, intensity of these dark pixels can directly deliver exact estimation of haze's transmission. Various enhancement and dehazing techniques based on dark channel [18], [19], [20], [21], [23] have been developed earlier by researchers to remove the haze and improve the quality of the images.

For an arbitrary image $\mathrm{J}(\mathrm{x})$, its dark channel is given by equation (1) [12] as

$$
\mathrm{J}^{\mathrm{dark}}(\mathrm{x})=\min _{\mathrm{c} \varepsilon\{\mathrm{r}, \mathrm{g}, \mathrm{b}\}}\left(\min _{\mathrm{y} \varepsilon \Omega(\mathrm{x})}\left(\mathrm{J}^{\mathrm{c}}(\mathrm{y})\right)\right)
$$

Where $\mathrm{J}^{\mathrm{c}}$ is a color channel of $\mathrm{J}(\mathrm{x})$ and $\Omega(\mathrm{x})$ is a local square patch centered on pixel $\mathrm{x}$.

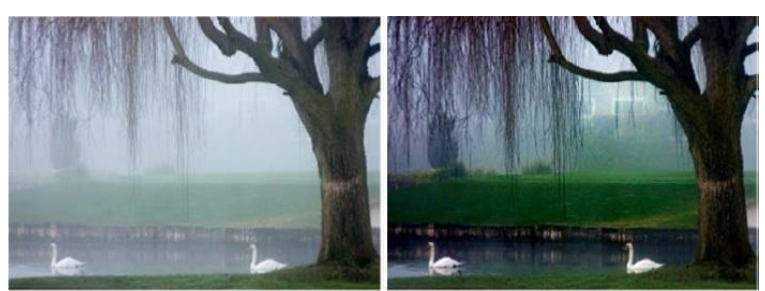

Figure 1 a) Input Image b) Result of Dark Channel Prior method [12]

\subsubsection{Limitations of Dark Channel Prior}

The dark channel prior may be invalid when the scene object is inherently similar to airlight over a large local region and no shadow is cast on the object [12].

\subsection{Fuzzy Logic}

The Fuzzy logic tool was introduced in 1965 by Lofti Zadeh. Fuzzy logic provides a good mathematical framework to deal with the uncertainty of information. Fuzzy set theory provides a capability to characterize the ambiguity and imprecision. Fuzzy logic is used to deal with multivalent kind of logic to improve the image enhancement techniques. Fuzzy logic is flexible as with any given system, it's easy to manage it [2].Fuzzy logic is conceptually easy to understand. The mathematical concepts behind fuzzy reasoning are very simple [2]. 


\subsection{Adaptive Histogram Equalization}

Adaptive histogram equalization (AHE) is a contrast enhancement method. It is useful for solving the uneven illumination problem in underwater images. It computes several histograms, each corresponding to a distinct section of the image and uses them to redistribute the lightness values of the image uniformly. It operates on small data regions called tiles, rather than the entire image. Each tile's contrast is enhanced, so that the histogram of the output region approximately matches the specified histogram. The neighboring tiles are then combined using bilinear interpolation in order to eliminate artificially induced boundaries. The size of the neighborhood region is a parameter of the method. It constitutes a characteristic length scale: contrast at smaller scales is enhanced, while contrast at larger scales is reduced [25]. The AHE process can be understood in different ways. In one perspective the histogram of grey levels (GL's) in a window around each pixel is generated first. The cumulative distribution of GL's, that is the cumulative sum over the histogram, is used to map the input pixel GL's to output GL's. If a pixel has a GL lower than all others in the surrounding window, the output is maximally black and if it has the median value in its window, the output is $50 \%$ grey [4].

\section{RELATED WORK}

S. M. Pizer et al. (1987) [1] described Adaptive histogram equalization (ahe) as a contrast enhancement which was broadly applicable and had demonstrated effectiveness. They also reported the various variations of adaptive histogram equalization such as full ahe, interpolated ahe, weighted ahe, clipped ahe to deal with different issues of adaptive histogram equalization. H.D. Cheng and H. Xu (2000) [3] In this paper, authors found that Fuzzy set theory was a useful tool for handling the uncertainty in the images associated with vagueness and/or imprecision. This paper presented a novel adaptive direct fuzzy contrast enhancement method based on the fuzzy entropy principle and fuzzy set theory. M. Hanmandlu, D. Jha and R. Sharma (2003) [6] This paper presented a Gaussian membership function that transformed the saturation and intensity histograms of HSV color model into the fuzzy domain. It introduced a new contrast intensification operator called NINT, which involves a parameter $t$ for enhancement of color images. The parameter $t$ was calculated globally by minimizing the fuzzy entropy of the image information. The fuzzifier and intensification parameters were evaluated automatically for the input color image by optimizing the contrast and entropy in the fuzzy domain. The intensification operation leads to enhancement. Y.Y. Schechner and N. Karpel (2005) [7] analyzed the physical effects of visibility degradation and proposed an image recovery algorithm based on a couple of images taken at different orientations with a polarizer which resulted in proposed an image recovery algorithm based on a couple of images taken at different orientations with a polarizer. $\mathbf{R}$. Fattal (2008) [9] presented a mathematical model for image dehazing. This model described the surface shading of the objects and the scene albedo. By assuming that the two functions were locally statistically uncorrelated, hazy images can be divided into regions of constant albedo. These can be used to infer the actual scene. N. Desai et al. (2009) [10] proposed a novel fuzzy logic based algorithm to de-weather fog degraded images. Airlight was estimated using fuzzy logic followed by color correction for enhanced visibility. K. He, J. Sun and X. Tang (2011) [12] proposed a method called dark channel prior. Dark Channel prior was a statistics of haze free outdoor images. It proposed that some pixels had low intensity in atleast one color channel. These pixels used in combination with haze imaging model estimated the thickness of haze. Soft matting had been done to refine the transmission. E. Ullah, R. Nawaz and J. Iqbal (2013)[16] presented a method which refined the existing dark channel prior further by considering chromatic and achromatic aspects of image and proposed dark channel model using the HIS color model. Dark channel proposed was the minimum of (1-S) and (I) components .So, transmission $\mathrm{t}$ was changed accordingly. Color vibrancy and contrast of restored images had been improved. K. He, J. Sun, and X. Tang (2013) [17] In this paper, authors presented a novel image filter called guided filter which was applicable in computer vision and graphics. The guided filter computed the filtering output by considering the content of a guidance image, which can be the input image itself or another different image. They designed a new filter that exhibits property of edge preserving smoothing but which can be computed efficiently and non-approximately. This filter was applicable for structure-transferring, enabling novel applications of filtering-based feathering/matting and dehazing. The guided filter had a common limitation that it may exhibit halos near some of the edges. G. Raju and M. S. Nair (2014) [22] proposed a novel fuzzy logic and histogram based algorithm for enhancing low contrast color images. This approach was mainly based in two important parameters $M$ and $K$ where $M$ was the average intensity value of the image and $\mathrm{K}$ was contrast intensification parameter. This approached used HSV color space where only V component was stretched by preserving the chromatic information such as hue $(\mathrm{H})$ and Saturation (S). The advantages of this method are computationally fast and improved visual quality of the images. The limitation of the approach was it can be only applied to low contrast and low bright color images. $\mathbf{S}$. Serikawa and H. Lu (2014) [24] proposed a fast joint trilateral filter for underwater image dehazing. A new underwater model is also proposed to compensate the attenuation discrepancy along the propagation path. The joint trilateral filter removes overly dark fields of underwater images by refining transmission depth map. The proposed joint trilateral filter also acts as edge preserving smoothing operator which had better behavior near the edges. The enhanced images are characterized by reduced noise level, improved quality, finest details and edges are enhanced, better exposedness of dark regions.

\section{PROPOSED WORK}

So, various techniques have been proposed in literature to remove the haze from underwater images. But still some problems exist. The images look dark after haze removal, this problem is neglected by most of the existing research. In most of the existing literature, the lower bound value for restoration is taken static as 0.1.So, we have proposed a new underwater image haze removal method that uses fuzzy logic on dark channel and adaptive histogram equalization to improve the effectiveness of haze removal and the quality of underwater image. In proposed methodology, first of all we input hazy underwater image. We find the dark channel and evaluate airlight in input image. Then dark channel of input image and transmission map is refined .After that, fuzzy logic is used to focus on the adaptability by computing optimized lower bound value for restoration in haze removal .Now, Adaptive Histogram Equalization is used to remove the problem of uneven illumination. Then image is restored using restoration model. As output, we get a final image that has haze removed to a great extent and quality of underwater image improved. 


\section{EPERIMENTAL RESULTS AND DISCUSSION}

In the experiments, the proposed technique has been implemented to test a wide variety of underwater images. The novel algorithm has been implemented in MATLAB. We tested our algorithm for many different foggy and hazy underwater images. It successfully removed the haze from underwater images to a great extent. In this section, first of all, we compare the existing technique joint trilateral filter [24] with our proposed technique fuzzy based DCP and AHE for underwater image haze removal by showing their comparison through images. Then both techniques are compared on the basis of values evaluated by various image performance metrics through tables and graphs.

\subsection{Results of Joint Trilateral Filter}

Fig. 2 and 3 shows the input images and corresponding results of the underwater image haze removal by Joint Trilateral Filter [24]. It is clearly shown that all the haze has been removed to a great extent but image look dark after haze removal. So we can say the given technique is not so good for solving the uneven illumination problem in underwater images and contrast enhancement needs to be done.

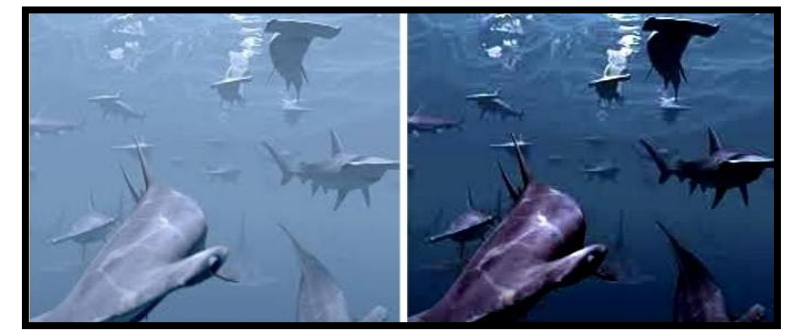

Figure 2 Input image \& results of Existing Technique

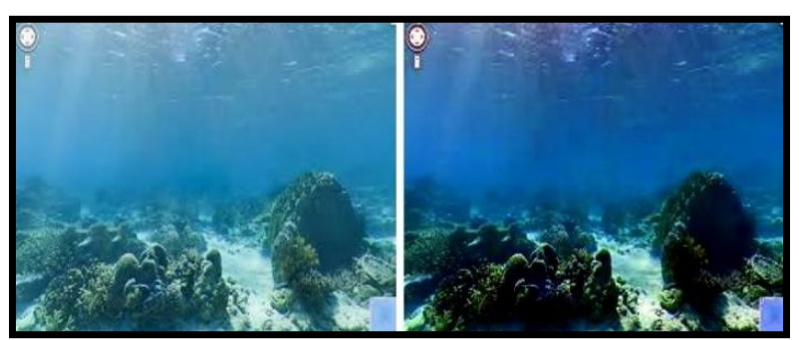

Figure 3 Input images and results of Existing Technique

\subsection{Results of Proposed Technique}

Fig. 4 and 5 shows the input images and corresponding results of underwater image haze removal by Fuzzy Based Dark Channel Prior and Adaptive Histogram Equalization. It is clearly shown that the proposed approach has successfully removed the haze to a great extent. The contrast has been improved and problem of uneven illumination has been sorted out.

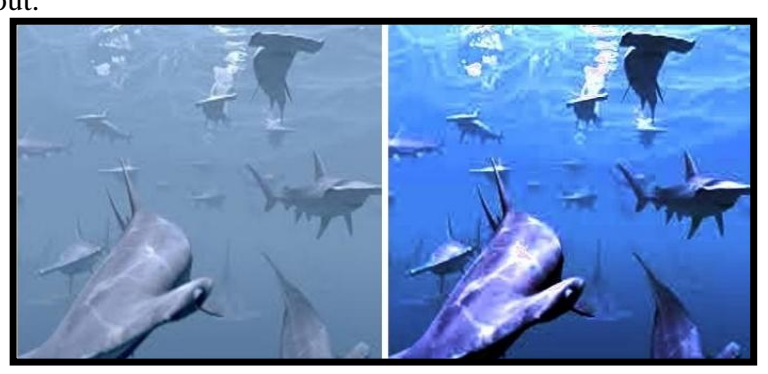

Figure 4 Input image and results of Fuzzy Based DCP and AHE

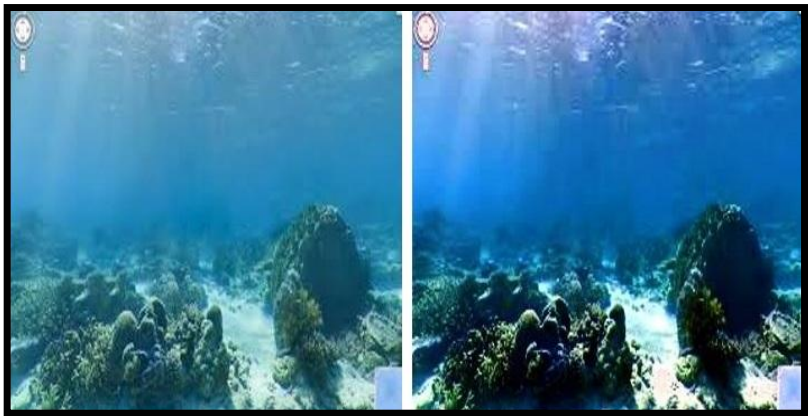

Figure 5 Input image and results of Fuzzy Based DCP and AHE

\section{PERFORMANCE EVALUATION}

This section contains the tabular and graphical comparison between existing [24] and proposed underwater haze removal algorithms based upon image quality evaluation metrics such as PSNR (Peak Signal to Noise Ratio), MSE (Mean Square Error) and RMSE (Root Mean Square Error).

\subsection{Mean Square Error}

Mean Square Error [26] is a risk function corresponding to expected value of the squared error or quadratic loss. The large value of Mean Square Error means the image is of poor quality. MSE is generally expressed as

$$
M S E=\frac{1}{m m} \sum_{i=0}^{m-1} \sum_{j=0}^{n-1}[I(i, j)-K(i, j)]^{2}
$$

Where $\mathrm{I}$ is the noise free $\mathrm{m} * \mathrm{n}$ monochrome image and $\mathrm{K}$ is the noisy approximation.

Table 1 and Fig. 6 has shown the comparison between existing technique and proposed technique of underwater image haze removal based on Mean Square Error (MSE).As MSE is less in almost every underwater image as a result of applying proposed technique, therefore this proposed technique has shown effective and better results over existing technique.

Table 1 Mean Square Error Analysis

\begin{tabular}{|c|c|c|}
\hline Image & Existing Technique & Proposed Technique \\
\hline IMG1 & 0.051 & 0.017 \\
\hline IMG2 & 0.047 & 0.006 \\
\hline IMG3 & 0.062 & 0.020 \\
\hline IMG4 & 0.069 & 0.032 \\
\hline IMG5 & 0.057 & 0.051 \\
\hline IMG6 & 0.017 & 0.010 \\
\hline IMG7 & 0.063 & 0.030 \\
\hline IMG8 & 0.064 & 0.052 \\
\hline IMG9 & 0.104 & 0.035 \\
\hline IMG10 & 0.031 & 0.014 \\
\hline
\end{tabular}


The graph obtained for MSE of these images is shown in fig. 6 below:

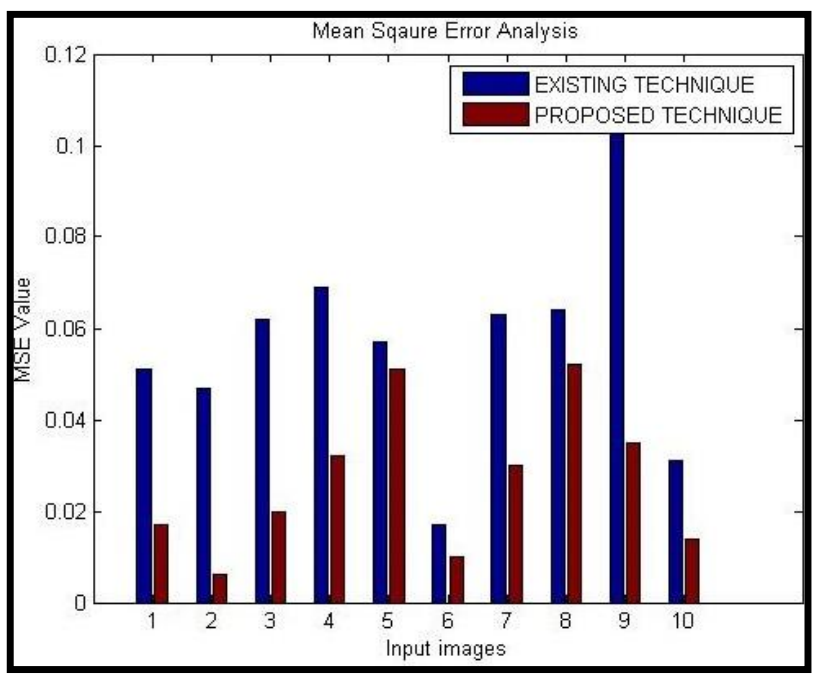

Figure 6 MSE Analysis

\subsection{Peak Signal to Noise Ratio}

Peak Signal-to-Noise ratio [27] is the ratio between the maximum possible power of a signal and the power of corrupting noise that affects the fidelity of its representation. As many signals have a wide dynamic range, PSNR is generally expressed in terms of logarithmic decibel scale.

$$
\mathrm{PSNR}=20 * \log _{10} \frac{\mathrm{MAX}}{\mathrm{MSE}}
$$

Where MAX is maximum possible pixel value of the image and MSE is Mean Square Error.

Table 2 and Fig. 7 has shown the comparison between existing technique and proposed technique of underwater image haze removal based on Peak Signal to Noise Ratio (PSNR).As PSNR is more in almost every underwater image after applying proposed underwater image haze removal technique, therefore proposed technique has shown significant enhanced and good results over existing technique.

Table 2 Peak Signal to Noise Ratio Analysis

\begin{tabular}{|c|c|c|}
\hline Image & Existing Technique & Proposed Technique \\
\hline IMG1 & 60.9 & 65.7 \\
\hline IMG2 & 61.3 & 69.8 \\
\hline IMG3 & 60.1 & 65.08 \\
\hline IMG4 & 59.7 & 63.02 \\
\hline IMG5 & 60.5 & 60.9 \\
\hline IMG6 & 65.6 & 67.9 \\
\hline IMG7 & 60.1 & 63.3 \\
\hline IMG8 & 60.01 & 60.8 \\
\hline IMG9 & 57.9 & 62.5 \\
\hline IMG10 & 63.1 & 66.6 \\
\hline
\end{tabular}

The graph obtained for PSNR of these images is shown in fig. 7 below:

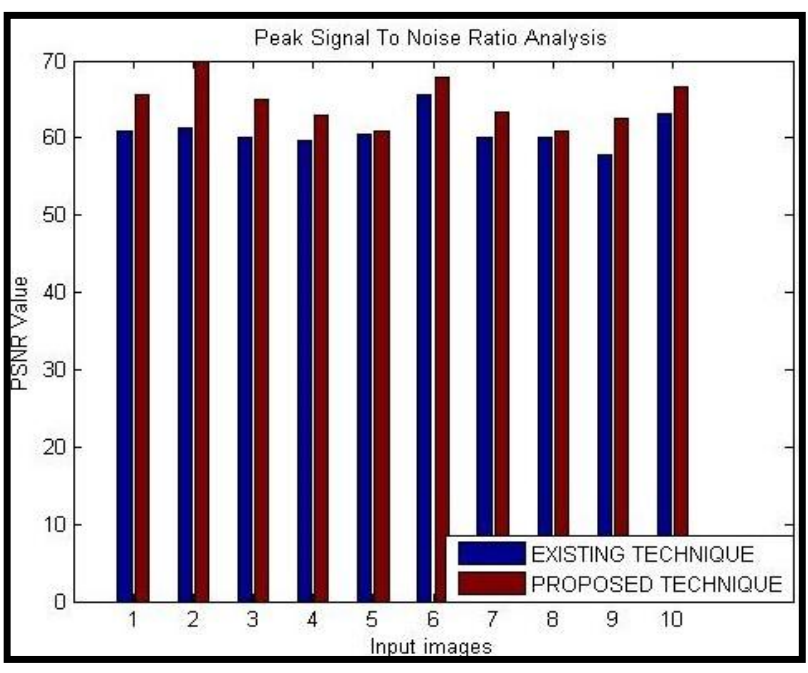

Figure 7 PSNR Analysis

\subsection{Root Mean Square}

Root Mean square error (RMSE) [28] is a measure of differences between values predicted by a model or an estimator and the values actually observed. Root mean square error (RMSE) is the square root of mean squared error (MSE).Basically, the RMSE represents the sample deviation of the differences between predicted and observed values. Root Mean square error value should be less for an image to be of good quality. Root Mean Square Error is computed as:

$$
\mathrm{RMSE}=\sqrt{\mathrm{MSE}}
$$

Table 3 and Fig. 8 has shown the comparison between existing technique [24] and proposed technique of underwater image haze removal based on Root Mean Square Error (RMSE).As RMSE is less in almost every underwater image after applying proposed technique, therefore technique has shown significant good results over existing technique.

Table 3 Root Mean Square Error Analysis

\begin{tabular}{|c|c|c|}
\hline Image & Existing Technique & Proposed Technique \\
\hline IMG1 & 0.227 & 0.131 \\
\hline IMG2 & 0.218 & 0.082 \\
\hline IMG3 & 0.249 & 0.141 \\
\hline IMG4 & 0.263 & 0.180 \\
\hline IMG5 & 0.239 & 0.227 \\
\hline IMG6 & 0.132 & 0.102 \\
\hline IMG7 & 0.251 & 0.174 \\
\hline IMG8 & 0.254 & 0.230 \\
\hline IMG9 & 0.323 & 0.189 \\
\hline IMG10 & 0.178 & 0.118 \\
\hline
\end{tabular}


The graph obtained for RMSE of these images is shown in fig. 8 below:

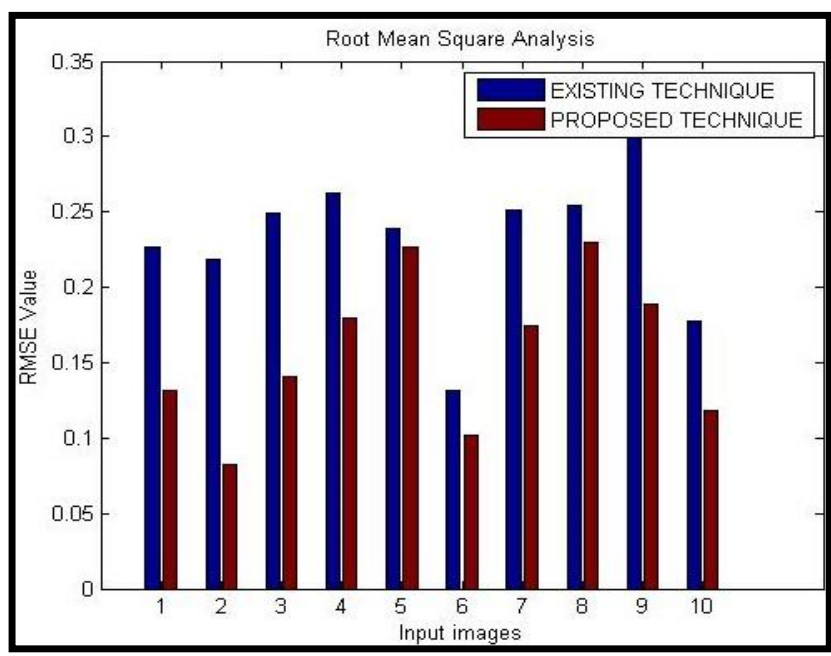

Figure 8 RMSE Analysis

\section{CONCLUSION}

Underwater image haze removal techniques have a very significant role in improving the quality and contrast of underwater images. Most of the existing techniques remove haze to a good extent but low contrast, darkness after haze removal and uneven illumination etc. problems still exist. This paper has proposed a new underwater image haze removal method to improve the effectiveness of haze removal and enhance quality of underwater images. The comparative analysis has shown that the proposed technique has better results than existing technique on the basis of results of visual analysis and performance metrics. In future, the proposed technique can also be applied on remote sensing images.

\section{REFERENCES}

[1] S. M. Pizer et al. "Adaptive histogram equalization and its variations" Computer vision, graphics, and image processing (Elsevier), 39, no.3, pp. 355-368, 1987.

[2] J. S Roger Jang and N. Gulley. "Fuzzy logic toolbox user's guide." The Mathworks Inc1, 1995.

[3] H .D Cheng, and H. Xu "A novel fuzzy logic approach to contrast enhancement." Pattern Recognition (Elsevier), 33, no. 5, pp.809-819, 2000.

[4] J. A. Stark "Adaptive image contrast enhancement using generalizations of histogram equalization." IEEE Transactions on Image Processing, 9, no. 5, pp. 889-896, 2000.

[5] J. Yuh and M. West "Underwater robotics." Advanced Robotics, 15, no. 5, pp. 609-639, 2001.

[6] M. Hanmandlu, D. Jha, and R. Sharma. "Color image enhancement by fuzzy intensification" Pattern Recognition Letters (Elsevier), 24, no. 1, pp. 81-87, 2003.

[7] Y.Y. Schechner, N. Karpel "Recovery of underwater visibility and structure by polarization analysis", IEEE J.ournal of Oceanic Engineering, 30, no. 3, pp.570-587, 2005.
[8] J. Heidemann , W. Ye, J. Wills,A. Syed \& Y. Li "Research challenges and applications for underwater sensor networking" In Wireless Communications and Networking Conference, IEEE, Vol. 1, pp. 228-235, 2006.

[9] R. Fattal "Single image dehazing." In ACM Transactions on Graphics (TOG), vol. 27, no. 3, 2008.

[10] N. Desai et al. "A Fuzzy Logic Based Approach to DeWeather Fog-Degraded Images." In IEEE Sixth International Conference on Computer Graphics, Imaging and Visualization, pp. 383-387, 2009.

[11] R. Schettini and S. Corchs. "Underwater image processing: state of the art of restoration and image enhancement methods." EURASIP Journal on Advances in Signal Processing, 2010.

[12] K. He, J. Sun, and X. Tang. "Single image haze removal using dark channel prior" IEEE Transactions on Pattern Analysis and Machine Intelligence, 33, no.12, pp. 23412353,2011

[13] H.Y. Yang, P.Y. Chen, C.C. Huang, Y.Z. Zhuang \& H.Y. Shiau "Low complexity underwater image enhancement based on dark channel prior." IEEE Second International Conference on Innovations in Bioinspired Computing and Applications, pp. 17-20, 2011.

[14] A.T. Çelebi \& S. Ertürk,"Visual enhancement of underwater images using Empirical Mode Decomposition" Expert Systems with Applications(Elsevier), 39(1), pp. 800-805, 2012.

[15] J.Y. Chiang and Y.C Chen. "Underwater image enhancement by wavelength compensation and dehazing." IEEE Transactions on Image Processing, 21, no. 4, pp. 1756-1769, 2012.

[16] E. Ullah, R. Nawaz and J. Iqbal. "Single image haze removal using improved dark channel prior." In Proceedings of IEEE International Conference on Modelling, Identification \& Control, pp. 245-248, 2013.

[17] K. He, J. Sun, and X. Tang, "Guided image filtering", IEEE Transactions on Pattern Analysis and Machine Intelligence, vol. 35, no. 6, pp. 1397-1409, June 2013.

[18] Q. Zhu, S. Yang, P. A. Heng \& X. Li "An adaptive and effective single image dehazing algorithm based on dark channel prior." In IEEE International Conference on Robotics and Biomimetics,pp. 1796-1800, 2013.

[19] Y. J. Cheng et al. "Visibility Enhancement of Single Hazy Images Using Hybrid Dark Channel Prior." In IEEE International Conference on Systems, Man, and Cybernetics, pp. 3627-3632, 2013.

[20] Z. Chen, J. Shen and P. Roth. "Single Image Defogging Algorithm based on Dark Channel Priority." Journal of Multimedia, 8, no. 4, pp. 432-438, 2013.

[21] A. Alajarmeh, R. A. Salam,M. F. Marhusin \& K. Abdulrahim "Real-time video enhancement for various weather conditions using dark channel and fuzzy logic." In IEEE International Conference on Computer and Information Sciences, pp. 1-6, 2014. 
[22] G. Raju and M. S. Nair. "A fast and efficient color image enhancement method based on fuzzy-logic and histogram." AEU-International Journal of Electronics and Communications (Elsevier), 68, no. 3, pp. 237-243, 2014.

[23] P. F. Chen, J. K. Guo, C.C. Sung \& H. H. Chang "An Improved Dark Channel-Based Algorithm for Underwater Image Restoration." In Advanced Materials Springer International Publishing, pp. 311-316, 2014.

[24] S. Serikawa and H. Lu "Underwater image dehazing using joint trilateral filter." Computers \& Electrical Engineering (Elsevier), 40, no. 1, pp. 41-50, 2014.
[25] "Adaptive Histogram Equalization”, [Online Available], http://www.wikipedia.com

[26] "Mean Squared Error", [Online Available], http://www.wikipedia.com

[27] "Peak signal to noise Ratio", [Online Available], http://www.wikipedia.com

[28] "Root mean square deviation", [Online Available], http://www.wikipedia.com 